\title{
Electrical Impedance Tomography: Monitoring of Pulmonary Ventilation in Pediatric Cardiac Postoperative
}

Carolina Vieira de Campos ${ }^{1}$, Paula Vieira Vincenzi Gaiolla ${ }^{1}$, Marcelo Bisegli Jatene ${ }^{1}$, Cintia Johnston ${ }^{1 *}$ and Werther Brunow de Carvalho ${ }^{1}$

${ }^{1}$ Pediatrics Department, Medical University of São Paulo, Brazil

\section{Introduction}

The electrical impedance tomography (EIT) is a bedside monitoring tool that noninvasively visualizes local ventilation and arguably lung perfusion distribution. Is a non-invasive radiation-free monitoring technique that provides images based on tissue electrical conductivity of the chest [1]. Some studies [2,3] including adult patients have applied EIT in the context of the preoperative, intraoperative period, extending to the postoperative follow-up of several clinical situations. However, so far there are no scientific publications in this context in the pediatric age group.

\section{Case}

We present the case of an 8-month-old girl with unbalanced atrioventricular septal defect, small right-ventricle and non-restrictive atrial and ventricular septal defects, status-post pulmonary artery (PA) banding at 3 months, who underwent a Superior Bilateral Vena Cava anastomosis, atrioventricular valve plasty and PA re-banding (total closure was not possible due to hypoxia). Her post-operative course was complicated by hypoxic spells which lead to a failed extubation and need to increase support through the first week postoperatively. Investigation took place and the hypoxemia was found to be multifactorial: ventricular dysfunction, mild stenosis on the Left Superior Vena Cava anastomosis, borderline PA pressure and atelectasis. In order to find the best ventilation strategy on such a complex case, an electrical impedance tomography (EIT), Enlight $1800^{\circ}$, (Timpel, Brazil) was installed and showed a decrease on left-lung ventilation (Figure 1). An increase in ventilation pressures was safely performed, with satisfactory left lung recruitment and no hemodynamic instability.

Bronchoscopy revealed a mild extrinsic compression on left main bronchus and angio-CT (Figure 2) ruled out malformations that needed to be addressed. After hemodynamic optimization with levosimendan

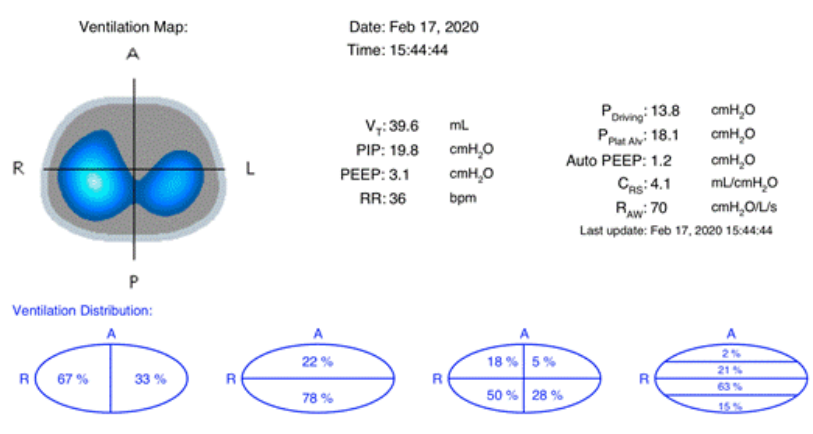

Figure 1. Electrical impedance tomography (EIT) showing reduced left lung ventilation

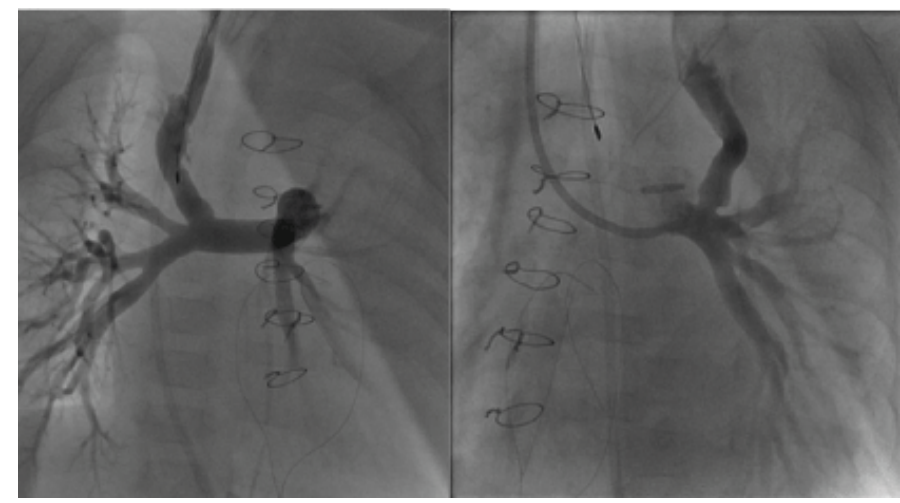

Figure 2. Invasive Angiography showing normal blood flow on the right superior vena cava anastomosis (above) and mild stenosis on the left superior vena cava anastomosis

and sildenafil, she was successfully weaned from support and extubated to a high-flow nasal cannula on postoperative day 18 . The rest of her clinical course was unremarkable.

The EIT can help safely finding the best ventilation strategy for patient with pulmonary blood flow dependent on intrathoracic pressures [4], for whom a delicate balance between lung ventilation and perfusion is mandatory for a good surgical outcome. The information obtained through EIT can be analyzed online and offline to detect recruitment, derecruitment, overdistension or variation of poorly ventilated lung units (silent spaces) in response to PEEP changes, and pendelluft phenomenon in spontaneously breathing patients [5]. In addition, with this technique it is also feasible to estimate the local lung perfusion and associating these data with the ventilation signal mapping of regional ventilation-perfusion ratios can be provided. Pulmonary conditions are constantly changing and continuous EIT monitoring can be a useful tool for detecting these variations [6].

EIT creates non-invasive and radiation-free images of the regional ventilation and lung perfusion distribution at the bedside. Functional EIT images allow different functional characteristics of the respiratory and cardio-circulatory system to be addressed. EIT can be combined with other signals, such as airway pressure, enabling the assessment of regional respiratory system compliance (CRS) or regional pressure/ volume $(\mathrm{P} / \mathrm{V})$ curves. Changes in lung ventilation and EELV,

${ }^{\star}$ Correspondence to: Cintia Johnston, Clinical Research Assistant Professor of Neonatology and Intensive Care, Pediatrics Department, Medical University of São Paulo - FMUSP, Brazil, E-mail: cintiajohnston@terra.com.br

Received: June 09, 2021; Accepted: June 17, 2021; Published: June 25, 2021 
compliance, CRS, P/V curves, as well as alveolar overdistension and collapse can be assessed by EIT dynamically on a regional level [7].

EIT has been shown useful for monitoring of regional lung perfusion as well as stroke volume estimation. Continuous and non-invasive perfusion monitoring can be performed based on the EIT waveforms resulting from cardiac activity in both the heart and lung region, whereas intermittent and invasive monitoring is possible using contrast-agents such as hypertonic saline injected through a central venous line $[7,8]$.

EIT is a promising technique for the perioperative assessment of surgical patients, providing tailored adaptive respiratory and haemodynamic monitoring [8]. Further studies are needed to address the current technological limitations, confirm the findings and evaluate which patients can benefit more from this technology.

\section{References}

1. Frerichs I, Amato MB, Van Kaam AH, Tingay DG, Zhao Z, et al. (2017) Chest electrical impedance tomography examination, data analysis, terminology, clinical use and recommendations: consensus statement of the TRanslational EIT developmeNt stuDy group. Thorax 72: 83-93. [Crossref]
2. Bauer M, Opitz A, Filser J, Jansen H, Meffert RH, et al. (2019) Perioperative redistribution of regional ventilation and pulmonary function: a prospective observational study in two cohorts of patients at risk for postoperative pulmonary complications. BMC Anesthesiol 19: 132. [Crossref]

3. Scaramuzzo G, Spadaro S, Waldmann AD, Böhm SH, Ragazzi R, et al. (2019) Heterogeneity of regional inflection points from pressure-volume curves assessed by electrical impedance tomography. Crit Care 23: 119. [Crossref]

4. Jang GY, Ayoub G, Kim YE, Oh TI, Chung CR, et al. (2019) Integrated EIT system for functional lung ventilation imaging. Biomed Eng Online 18: 83. [Crossref]

5. Tomicic V, Cornejo R (2019) Lung monitoring with electrical impedance tomography: technical considerations and clinical applications. J Thorac Dis 11: 3122-3135. [Crossref]

6. Brunow de Carvalho W, Fonseca MC, Johnston C (2007) Electric impedance tomography, the final frontier is close: the bedside reality. Crit Care Med 35: 19961997. [Crossref]

7. Putensen C, Hentze B, Muenster S, Muders T (2019) Electrical Impedance Tomography for Cardio-Pulmonary Monitoring. J Clin Med 8: 1176. [Crossref]

8. Spinelli E, Mauri T, Fogagnolo A, Scaramuzzo G, Rundo A, et al. (2019) Electrical impedance tomography in perioperative medicine: careful respiratory monitoring for tailored interventions. BMC Anesthesiol 19: 140. [Crossref]

Copyright: (C2021 De Campos CV. This is an open-access article distributed under the terms of the Creative Commons Attribution License, which permits unrestricted use, distribution, and reproduction in any medium, provided the original author and source are credited. 